\title{
ESTUDIOS EPIDEMIOLÓGICOS EN ÁREAS PEQUEÑAS: HERRAMIENTAS PARA ANALIZAR LA CONTAMINACIÓN AMBIENTAL Y SUS EFECTOS EN SALUD A ESCALA LOCAL
}

\author{
Nelson E. Arias-Ortiz ${ }^{\underline{1}}$ \\ Recibido el 24 de julio de 2014, aprobado el 23 de marzo de 2015 y \\ actualizado el 11 de noviembre de 2015
}

DOI: 10.17151/luaz.2016.42.20

\section{RESUMEN}

La creciente contaminación de origen antrópico ha generado preocupación mundial por sus potenciales efectos perjudiciales para la salud humana. Ante esta necesidad de investigación, los estudios epidemiológicos espaciales en áreas pequeñas se presentan como un abordaje sencillo y eficiente, que utiliza datos recopilados de manera rutinaria para analizarlos mediante técnicas estadísticas sofisticadas, ofreciendo herramientas para estudiar asociaciones epidemiológicas a escala local. En este trabajo se presenta una revisión de las generalidades y los elementos metodológicos y estadísticos de estos estudios, con algunos ejemplos de estudios publicados en las dos últimas décadas, y se discuten algunos puntos relevantes.

\section{PALABRAS CLAVE}

Epidemiología, análisis espacial, contaminación, áreas pequeñas.

\section{EPIDEMIOLOGICAL STUDIES IN SMALL AREAS: TOOLS FOR ANALYZING ENVIRONMENTAL POLLUTION AND ITS HEALTH EFFECTS ON LOCAL SCALE}

\begin{abstract}
The increasing anthropogenic pollution has generated worldwide concern about potential adverse effects on human health. Given this need for research, spatial epidemiological studies in small areas are presented as a simple and efficient approach, using routinely collected data to be analyzed using sophisticated statistical techniques, providing tools to study locally epidemiological associations. A review of generalities and methodological and statistical elements of these studies is presented in this article with some examples of studies published in the last two decades, and a brief discussion about some relevant issues.
\end{abstract}

KEY WORDS: Epidemiology, spatial analysis, pollution, small areas.

\section{INTRODUCCIÓN}

En las últimas décadas ha habido una creciente preocupación por los efectos en salud que pueden tener los contaminantes ambientales (Morris y Wakefield, 2000), en especial por el amplio número de sustancias químicas que utiliza la industria moderna, muchas de las cuales son 
tóxicas para la salud humana, incluyendo varias sustancias con reconocidos efectos carcinogénicos (Belpomme et al., 2007; Boyle y Levin, 2008; Cogliano et al., 2011).

Existen dos escenarios de investigación de los efectos en salud potencialmente asociados a fuentes de contaminación. El primero -y más común- ocurre cuando las comunidades, los medios, o los profesionales de la salud llaman la atención sobre un posible exceso de casos en un área determinada y proponen una asociación entre el exceso de casos y la presencia de una fuente contaminante, o análisis post hoc (Wilkinson y Armstrong, 2006). Ante una alarma por un aparente exceso de casos, las autoridades sanitarias están en la obligación de dar una respuesta fundamentada, lo que justifica la investigación de posibles conglomerados mediante estudios en etapas, como lo sugieren los protocolos de los centros para la prevención y control de las enfermedades de los Estados Unidos (Centers for Disease Control and Prevention, 1990).

El segundo escenario se da cuando se considera a priori, es decir, sin conocer la distribución espacial de los casos, que una o varias fuentes de contaminación afectan el patrón de ocurrencia de los casos sobre la base de una hipótesis específica y biológicamente plausible, o en ausencia de hipótesis precisas a manera de análisis exploratorio en investigación etiológica. Particularmente en este segundo escenario, los estudios epidemiológicos en áreas pequeñas ofrecen una interesante opción metodológica para aproximarse al estudio de efectos en salud asociados a la contaminación a escala local (Wilkinson y Armstrong, 2006).

En la primera sección de este trabajo se plantean algunas generalidades de estos estudios. En la segunda parte se tratan elementos metodológicos y estadísticos. En la tercera, se presentan algunos ejemplos de estudios que hacen uso de los conceptos de la epidemiología espacial para establecer asociaciones entre contaminación y efectos en salud en áreas pequeñas $\mathrm{y}$, al final, se discuten algunos puntos relevantes.

\section{GENERALIDADES DE LOS ESTUDIOS EPIDEMIOLÓGICOS SOBRE CONTAMINACIÓN EN ÁREAS PEQUEÑAS}

\section{Contaminación ambiental y epidemiología espacial}

El estudio de exposiciones ambientales y sus efectos en salud ha sido fortalecido por el desarrollo de técnicas analíticas espaciales y por el avance de la tecnología computacional y de los sistemas de información geográfica (Beale, Abellán, Hodgson y Jarup, 2008), y hacen parte de un campo de estudio conocido como 'Epidemiología Espacial'.

La epidemiología espacial hace referencia a "la descripción y análisis de datos de salud geográficamente indexados, en relación con factores de riesgo ambientales, demográficos, socioeconómicos, comportamentales, genéticos o infecciosos" (Elliott y Wartenberg, 2004, p. 998), e involucra un espectro de metodologías que van desde lo puramente descriptivo hasta aproximaciones que buscan probar hipótesis.

Los diseños ecológicos en epidemiología ambiental son abordajes sencillos y eficientes, porque se ajustan al tipo de datos en salud disponibles, como son las estadísticas de incidencia y mortalidad, y a los datos sobre las exposiciones a contaminantes ambientales que "[...] 
necesariamente son medidas en el nivel de grupo, porque los datos a nivel individual no están usualmente disponibles y su recolección es poco práctica" (Rothman, Greenland y Lash, 2008, p. 99)².

La creciente disponibilidad de datos y los avances en las técnicas de análisis han favorecido la realización de estudios ecológicos en 'áreas pequeñas' (distritos censales, o zonas postales, por ejemplo), que mejoran los tradicionales reportes epidemiológicos a escalas nacionales 0 regionales, y facilitan el estudio de exposiciones a fuentes de contaminación a escala local, minimizando el sesgo ecológico en la medida en que las estimaciones locales son más cercanas a las estimaciones individuales, y permiten probar y seleccionar hipótesis sobre asociaciones epidemiológicas que posteriormente puedan validarse y comprobarse a nivel individual (Elliott y Wartenberg, 2004).

\section{Fuentes de contaminación ambiental}

Con respecto a la contaminación atmosférica, las fuentes se clasifican en cuatro categorías: fuentes fijas o puntuales, fuentes dispersas o de área, fuentes móviles, y fuentes naturales (INE-SERMANAT, 2005). En lo que se refiere al agua y al suelo, las principales fuentes de contaminación incluyen fuentes puntuales, como los vertimientos domésticos e industriales, y fuentes no puntuales como los escurrimientos agrícolas y urbanos (Nazaroff y Álvarez-Cohen, 2001), así como la presencia natural de sustancias potencialmente tóxicas para los humanos como el plomo y el arsénico, entre otros metales.

Desde un punto de vista práctico, en los estudios espaciales en áreas pequeñas suelen considerarse dos tipos de fuentes de contaminación, de acuerdo con su configuración espacial: i) fuentes puntuales como industrias, minas, incineradores, centrales eléctricas, o rellenos sanitarios; y ii) fuentes lineales como ríos, autopistas, vías férreas o líneas de transporte de electricidad (Morris y Wakefield, 2000).

Los asentamientos industriales han llamado particularmente la atención, en especial aquellas actividades industriales que emiten grandes cantidades de contaminantes al suelo, al aire o al agua. Entre los principales contaminantes emitidos por las industrias destacan el material particulado con diámetros aerodinámicos menores a 10 micrómetros $\left(\mathrm{PM}_{10}\right)$ y menores a 2,5 micrómetros $\left(\mathrm{PM}_{2,5}\right)$, las partículas ultra finas (PUF), los óxidos de azufre (SOx) y de nitrógeno (NOx), el monóxido (CO) y el dióxido de carbono $\left(\mathrm{CO}_{2}\right)$, el ozono $\left(\mathrm{O}_{3}\right)$, los compuestos orgánicos volátiles (COV), los hidrocarburos aromáticos policíclicos (HAP), las dioxinas, los furanos, los bifenilos policlorados y polibromados (PCB y PBB, por sus siglas en inglés, respectivamente) y algunos metales como arsénico, cadmio, mercurio, plomo, níquel, y cromo, entre otros (GarcíaPérez et al., 2007).

\section{Datos sobre contaminantes ambientales}

Según la Oficina de Rendición de Cuentas del Gobierno de los Estados Unidos, la Agencia de Protección Ambiental de ese país (EPA, por su sigla en inglés) ha podido ejercer sus facultades jurídicas exigiendo a la industria química pruebas de toxicidad de menos de 200 de las 62.000 sustancias químicas que se encontraban en el comercio en 1979. Más recientemente, la EPA ha podido obtener datos toxicológicos para 2.800 sustancias químicas mediante declaración voluntaria por parte de las 
industrias (United States Government Accountability Office, 2005). Sin embargo, aquellas se han negado a presentar pruebas para más de 300 sustancias identificadas en el programa de altos volúmenes de producción. Además, gran parte de la información es declarada por las industrias como confidencial, limitando directamente las acciones de los organismos de control (United States Government Accountability Office, 2005).

La medición válida y precisa de exposiciones ambientales es compleja y la información disponible con frecuencia es escasa (Elliott y Wartenberg, 2004). Varias razones podrían explicar la escasez de datos sobre exposiciones ambientales: primero, porque es imposible diseñar y conducir experimentos en humanos debido a implicaciones éticas obvias; segundo, porque la identificación de tóxicos, la cuantificación de relaciones dosis-respuesta, y la definición de límites máximos permisibles son procesos técnicamente difíciles; tercero, porque la exposición con frecuencia involucra mezclas de contaminantes en diversos ambientes, cuyas rutas de exposición pueden ser múltiples y con intensidad y duración diferentes; y cuarto, por los altos costos propios de las mediciones toxicológicas (Elliott y Wartenberg, 2004; United States Government Accountability Office, 2005; Boyle y Levin, 2008).

A diferencia de los estudios ocupacionales, donde las mediciones personales de exposición son el fundamento de las investigaciones, la medición de la exposición en epidemiología ambiental recae sobre el modelamiento de datos agregados, debido al mayor tamaño de las poblaciones y a diferencias en la naturaleza y distribución espaciotemporal de las exposiciones (Colvile, Briggs y Nieuwenhuijsen, 2003). La medición de exposiciones personales en estudios epidemiológicos de base poblacional es poco factible por las razones antes mencionadas.

Briggs (2000) describe cinco métodos de medición de exposición a contaminantes según su posición en la cadena que va desde el procesamiento y la emisión de los tóxicos en la fuente hasta la aparición de efectos en salud:

i) Los métodos de indicadores.

ii) Los modelos de dispersión.

iii) El monitoreo de contaminación.

iv) El monitoreo de microambientes.

v) El monitoreo personal.

Los métodos de indicadores se aplican en etapas iniciales del proceso y se basan en datos de las actividades industriales y de emisiones en la fuente. Los modelos de dispersión y el monitoreo de contaminación informan sobre el comportamiento de los contaminantes una vez son emitidos por la fuente. El monitoreo de microambientes mide la exposición a contaminantes en ambientes específicos, como por ejemplo, puestos de trabajo o espacios confinados. El monitoreo personal mide directamente la exposición a la que están sometidos los individuos, y puede incluir biomarcadores (Briggs, 2000).

Muchos estudios utilizan los métodos de indicadores pese a que existen métodos de medición de exposición más precisos, principalmente porque son fácilmente calculables y de muy bajo costo. Sin embargo, son limitados porque solo proveen medidas semicuantitativas y crudas de la exposición, y son poco específicos (Briggs, 2000). 
Las mediciones ambientales y los modelos de exposición pueden subestimar o sobreestimar la exposición personal debido a que no toman en cuenta las posibles diferencias en la ingestión, la inhalación y las tasas de absorción dérmica, ni las diferencias en las rutas y mecanismos de exposición entre los sujetos. No obstante, todavía puede haber una buena correlación entre las estimaciones de exposición ambiental y personal (Colvile et al., 2003). Para el caso de la contaminación del aire, algunos autores consideran que los modelos de exposición más refinados permiten obtener estimaciones de asociación más robustas que los modelos simplificados (Sahsuvaroglu et al., 2009).

\section{Registros sistemáticos de emisiones de contaminantes}

En países desarrollados se han implementado programas para mejorar la disponibilidad de datos públicos sobre contaminantes ambientales desde hace más de tres décadas. En EE.UU., el Inventario de Emisiones Tóxicas (TRI, por su sigla en Inglés) -un programa de obligatorio cumplimiento para las industrias en sectores como manufactura, minería de metales, generación de energía eléctrica, manufacturas químicas y tratamiento de residuos peligrosos- monitoriza el manejo de cerca de 650 tóxicos relacionados con cáncer $u$ otros efectos crónicos en salud humana, con efectos adversos agudos significativos para la salud humana, o con efectos adversos significativos sobre el ambiente (EPA, 2013).

Por su parte, el Registro Europeo de Emisiones y Transferencias de Contaminantes provee información sobre más de 30.000 establecimientos industriales en los Estados miembros de la Unión Europea más Islandia, Liechtenstein, Noruega, Serbia y Suiza; monitoriza 91 contaminantes clave entre los que se cuentan dioxinas, gases de efecto invernadero, metales pesados, pesticidas y sustancias orgánicas cloradas, en 65 actividades económicas pertenecientes a 9 sectores industriales: energía, producción y transformación de metales, industrias minerales, industria química, gestión de residuos y aguas residuales, fabricación y transformación de papel y madera, ganadería y acuicultura intensiva, productos de origen animal y vegetal de la industria alimentaria y de las bebidas, y otras actividades (Agencia Europea de Medio Ambiente, 2013).

De acuerdo con la Organización de Estados Americanos (OEA), México y Chile son los únicos países en América Latina y el Caribe que cuentan con Registro de Emisión y Transferencia de Contaminantes (RETC) desde mediados de la década pasada (Salinas, 2007). En el caso de Chile, desde 2005 su RETC monitoriza 80 parámetros con indicadores anuales agregados a nivel comunal (municipal) y con coordenadas para las fuentes fijas; entre los contaminantes reportados se encuentran material particulado, compuestos orgánicos volátiles, nitrógeno amoniacal, mercurio, monóxido de carbono, óxidos de nitrógeno, aceites y grasas, dibenzoparadioxinas, dióxido de carbono, sólidos suspendidos totales, fósforo total, demanda biológica de oxígeno, plomo, tolueno, dióxido de azufre, arsénico y benceno (Cortés y Qüense, 2014).

Desde 2005, Colombia creó el marco jurídico para la gestión de residuos peligrosos en el país, como parte de sus compromisos internacionales (Protocolo de Montreal, al Convenio de Basilea y Convenio de Estocolmo). Desde 2007 se puso en marcha el Registro de Generadores de Residuos Peligrosos que obliga a los establecimientos industriales y comerciales a inscribirse en el registro y a reportar los residuos generados anualmente, 
excepto aquellos establecimientos que generen menos de $10 \mathrm{~kg} / \mathrm{mes}$ de residuos. La información es autorreportada por los generadores a la autoridad ambiental regional (Corporaciones Autónomas Regionales en los departamentos y distritos), y de allí al IDEAM donde se publican los consolidados anuales para cada corporación (Ministerio de Ambiente y Desarrollo Sostenible, 2014). Este registro monitoriza 45 categorías de desechos y 4 grupos o corrientes de residuos (desechos metálicos o que contengan metales, desechos inorgánicos que puedan contener metales o materia orgánica, desechos orgánicos que puedan contener metales y materia inorgánica, y desechos que puedan tener constituyentes orgánicos o inorgánicos), de acuerdo con lo establecido en el Convenio de Basilea (Ministerio de Ambiente Vivienda y Desarrollo Territorial, 2005). Además de la información sobre residuos peligrosos, el Sistema de Información de Calidad del Aire -SISAIRE-, ofrece información pública sobre los principales contaminantes atmosféricos (material particulado, óxidos de azufre, óxidos de nitrógeno, ozono, monóxido de carbono, hidrocarburos metánicos y no metánicos) (Ministerio de Ambiente Vivienda y Desarrollo Territorial, 2010), aunque algunas de las estaciones instaladas en el país solo monitorizan algunos de ellos, y el número de estaciones es insuficiente para estimar concentraciones de contaminantes en áreas pequeñas. También se han realizado inventarios de emisiones atmosféricas en algunas ciudades capitales (Correal-Arboleda y GamboaSalazar, 2006; Peñaloza, 2010; Universidad Pontificia Bolivariana, 2010).

No obstante, estos registros no cubren un importante número de sustancias potencialmente tóxicas, ni cubren todas las actividades económicas. Además, no todas las industrias están obligadas a reportar datos, sino solo aquellas que cumplen ciertos criterios de capacidad instalada o que superan algunos umbrales de volumen de sustancias específicas, como es el caso del programa de 'grandes volúmenes de producción' en EE.UU. (United States Government Accountability Office, 2005).

\section{Fuentes de datos en salud: datos agregados y puntuales}

De acuerdo con Waller y Gotway (2004), las fuentes de datos en salud se clasifican en cuatro grupos: estadísticas vitales, reportes de eventos de notificación obligatoria, registros de enfermedades, y encuestas de salud. Algunos de estos datos son recopilados de manera rutinaria por los gobiernos a través de las autoridades sanitarias en diferentes niveles territoriales, y son de carácter público, aunque no siempre son de fácil acceso. Tales datos pueden ser puntuales o conteos agregados en áreas. Los datos puntuales suelen corresponder a direcciones de residencia o sitio de trabajo habituales al momento del diagnóstico o de la defunción, o reconstrucciones del historial habitacional. Los datos agregados corresponden usualmente a reportes de tasas a nivel de áreas geográficas mayores como departamentos (provincias, regiones, estados) o municipios.

Para los datos agregados, la escala o "granularidad" geográfica es un factor determinante del valor de las técnicas espaciales para estudiar factores de riesgo que están ampliamente distribuidos y varían entre áreas geográficas, como ocurre con las exposiciones ambientales (Cuzick y Elliott, 1992).

Si bien los datos puntuales aportan mayor información que los datos de conteo, los primeros rara vez se encuentran disponibles. En casi todos los 
países, las legislaciones consagran la protección de la información personal, por lo que la única información disponible sobre eventos en salud (y también sobre exposiciones y población en riesgo) consiste en datos agregados, con diferencias en la granularidad de los datos entre un país y otro. Al igual que ocurre con los datos sobre exposiciones ambientales, los datos agregados sobre desenlaces en salud deben analizarse a nivel ecológico con las consecuentes limitaciones como el sesgo ecológico y el uso de límites político-administrativos relativamente arbitrarios, cuya escala en ocasiones no es apropiada para la evaluación de exposiciones ambientales (Morris y Wakefield, 2000).

En Colombia, el Sistema Integral de Información de la Protección Social SISPRO- ofrece información epidemiológica agregada a nivel municipal y departamental sobre algunos desenlaces en salud, más específicamente, las prestaciones realizadas dentro del Sistema de Seguridad Social en Salud, en cuatro categorías: consultas ambulatorias, procedimientos, hospitalizaciones y consultas a los servicios de urgencias. También ofrece información consolidada sobre el aseguramiento en salud de la población, desagregada hasta el nivel municipal. En el corto plazo este sistema contará además con un módulo de información ambiental, que permitirá monitorizar y analizar desenlaces en salud en función de variables ambientales (Ministerio de Salud, 2015). Sin embargo, en la actualidad este sistema de información no cuenta con información pública desagregada en áreas geográficas más pequeñas que el nivel municipal.

\section{ASPECTOS METODOLÓGICOS Y ESTADÍSTICOS DE LOS ESTUDIOS EN ÁREAS PEQUEÑAS}

\section{Definición del área de análisis}

El tamaño del área y la población de estudio debe ser lo suficientemente grande para contener los efectos de la contaminación y para dar un contraste de la exposición en la población manteniendo constantes otros factores, pero no tan grande que involucre numerosas fuentes o que introduzca grandes diferencias en la población como, por ejemplo, diferencias en la urbanización (Morris y Wakefield, 2000), ni tan pequeño que genere un exceso de casos artificial, a lo que se le conoce como "adelgazamiento de límites" (Olsen, Martuzzi y Elliott, 1996). Krieger et al. (2002) sugieren el uso de áreas con población promedio desde 1.000 (equivalentes a los 'census block groups' en EE.UU.) hasta 4.000 habitantes (equivalentes a los 'census tracks' o distritos censales en los EE.UU.). Estudios españoles utilizan áreas pequeñas urbanas con población promedio cercana a los 1.000 habitantes (Puigpinós-Riera et al., 2011). Definiciones más clásicas señalan como área pequeña aquella donde ocurren menos de 20 casos de un determinado evento en salud (Elliott, 1995).

\section{Temporalidad}

Es necesario definir un período de tiempo de estudio, el cual debe considerar un período de latencia entre la potencial exposición y la aparición del evento, que varía según la enfermedad en estudio. Los diseños que incorporan períodos "antes" y "después" - del inicio de operaciones de una fuente de contaminación industrial, por ejemplotienen la ventaja de que la población actúa como su propio control, ajustando por factores de confusión (Morris y Wakefield, 2000), como la 
estructura y dinámica poblacional por edad y sexo, otras exposiciones ambientales, o condiciones socioeconómicas o de los servicios de salud que hayan podido cambiar durante los períodos de análisis. Cuando los desenlaces en estudio tienen períodos de latencia prolongados es ideal incorporar datos sobre la movilidad de los individuos, dependiendo de la disponibilidad de tales datos, de modo que las variaciones en las exposiciones ambientales puedan ser adecuadamente representadas (Hystad et al., 2012; Wheeler, Ward y Waller, 2012).

\section{Diseño de comparación cerca versus referencia}

Corresponde al diseño más simple para realizar una exploración inicial de datos agregados, en el cual se compara el riesgo en la población "expuesta" -por ejemplo, un barrio ubicado a cierta distancia de la fuente contaminante- con el riesgo en un área de referencia, que puede ser el riesgo global en la región o municipio al que pertenece la población expuesta. En este diseño los aspectos cruciales son la elección de la población expuesta y la disponibilidad de datos sobre variables confusoras (Morris y Wakefield, 2000), como edad, sexo, nivel socioeconómico, o prevalencia de factores de riesgo como tabaquismo, obesidad o susceptibilidad genética, por ejemplo.

\section{Diseño de comparación cerca versus lejos}

La región en estudio es dividida en una o más áreas "cercanas" expuestas-y una o más áreas "lejanas" -no expuestas-. Se comparan las razones de morbilidad/mortalidad estandarizadas (RME) - $u$ otras medidas estandarizadas de ocurrencia de eventos en salud como la incidencia acumulada, de acuerdo con el tipo de datos disponibles- de cada área mediante una razón, lo cual permite obtener el riesgo relativo del área expuesta con respecto al área no expuesta (Guajardo y Oyana, 2009). Para datos puntuales tipo caso-control, la condición de exposición igualmente es considerada positiva si el sitio de residencia del caso o del control cae dentro del área "cercana", y negativa si se ubica en el área "lejana"; se construyen tablas de $2 \times 2$ para cada estrato y se estiman Odds Ratio de exposición para los casos en comparación con los controles.

Este diseño asume que existe un gradiente de exposición en función de la distancia con respecto a la fuente de contaminación. Mediante procedimientos estadísticos se busca estimar un posible gradiente de efectos en salud igualmente en función de la distancia. Así, por ejemplo, si se encuentra un gradiente de incidencia que responde a un gradiente de exposición, se plantea una posible asociación causal que fundamenta estudios con mediciones individuales o, incluso, intervenciones para el control de las emisiones desde un enfoque precautorio (Elliott et al., 1996; Edwards et al., 2006; Nuvolone et al., 2011).

\section{Aspectos estadísticos: modelo Poisson para datos agregados}

La formulación básica para analizar conteos de eventos en áreas pequeñas está dada por la distribución de Poisson (Morris y Wakefield, 2000; Holowaty, Norwood, Wanigaratne, Abellán y Beale, 2010). En cada área $i$ se tiene una población en riesgo para un estrato ${ }^{\frac{3}{j}} j, \mathrm{Nij}$, y un número de casos observado, Yij. Si el evento en estudio es raro y no es una enfermedad infectocontagiosa, los conteos de casos en cada área pueden ser considerados como variables Poisson aleatorias e independientes: 


$$
Y_{i j} \sim P o\left(N_{i j} p_{i j}\right)
$$

donde es la probabilidad de enfermedad en la región $i$ y en el estrato $j$. Como es esperable cuando se trabaja con datos en áreas pequeñas (distritos censales, barrios, manzanas, etc.), el número de casos en cada área será insuficiente para estimar la probabilidad de enfermar en cada estrato en cada área; por ello se hace necesario asumir proporcionalidad de la forma:

$$
p_{i j}=q_{j} * \theta_{i}
$$

(Ecuación 2)

donde $q_{i}$ corresponde a un conjunto de probabilidades referencia de enfermedad en el estrato $j(j=1, \ldots, J)$, y es el riesgo relativo del área $i$. Estas probabilidades de referencia pueden ser estimadas para la totalidad del área en estudio (estandarización interna), o pueden ser obtenidas de un área mayor a la que pertenece el área de estudio como la región o el país (estandarización externa). Bajo la distribución Poisson, se tiene que:

$$
Y_{i}=\sum_{j=1}^{J} Y_{i j} \sim P o\left(E_{i} \theta_{i}\right),
$$

donde $E_{i}$ es el número de casos esperado en el área $i$, que se obtiene a partir de

$$
E_{i}=\sum_{j} N_{i j} * q_{j}
$$

Finalmente,

$$
\widehat{\theta}_{i}=Y_{i} / E_{i}
$$

es la Razón de Morbilidad Estandarizada (RME), que equivale al estimador máximo verosímil del riesgo relativo en el área $i$.

En ausencia de datos precisos sobre exposiciones a contaminantes ambientales, es conveniente considerar modelos de exposición con base en variables sustitutas (Lawson, Browne y Vidal Rodeiro, 2003; Sahsuvaroglu et al., 2009), como por ejemplo:

i) La presencia o ausencia de fuentes contaminantes dentro del área de análisis.

ii) La distancia a la fuente, medida usualmente en $\mathrm{km}$.

iii) La dirección con respecto a la fuente.

iv) La interacción distancia-dirección.

v) Modelos de dispersión complejos, que incorporan información meteorológica o hidrológica.

Un modelo general de riesgo/distancia asume la forma: 


$$
\theta_{i}=\rho\left\{1+g\left(d_{i}\right)\right\}
$$

donde es el riesgo de base en el área $i$, y es una función de la distancia que tiende a 0 cuando la distancia tiende a infinito. La función puede tomar varias formas de acuerdo con los modelos considerados para evaluar la exposición (Morris y Wakefield, 2000).

Dado que la RME puede ser muy inestable por tratarse de un evento raro y de poblaciones pequeñas, es necesario "estabilizar" los riesgos relativos mediante técnicas de suavizado, como los modelos jerárquicos (Olsen et al., 1996).

\section{Suavizado de estimaciones en áreas pequeñas: el Modelo Besag- York-Mollié (BYM)}

Se trata de un modelo jerárquico bayesiano, en el que el primer nivel corresponde al conteo de eventos, que se asume sigue una distribución Poisson condicionalmente independiente, como se explicó arriba. En el segundo nivel, el modelo asume la forma:

$$
\log \theta_{i}=\alpha+v_{i}+\varepsilon_{i}
$$

Donde es el logaritmo del riesgo relativo de base (intercepto); representa un efecto aleatorio de correlación espacialmente estructurada, que usualmente es definido como una correlación de forma condicional autorregresiva (CAR, por sus sigla en inglés) que describe la variación espacial de factores de riesgo -medidos y no medidos- y se basa en que las áreas vecinas tienden a tener factores de riesgo similares; y $E_{i}$ corresponde a la variabilidad extra-Poisson no estructurada espacialmente (Lawson et al., 2003; Bilancia y Fedespina, 2009). El tercer nivel corresponde a las distribuciones de probabilidad a priori para los parámetros de los efectos aleatorios definidos en el segundo nivel, que habitualmente corresponden a una distribución Gamma, con valor esperado $a / b$, y varianza $a / b^{2}$, la cual puede adoptar diferentes valores en sus parámetros, de acuerdo con el conocimiento previo que se desee incorporar al modelo, siendo frecuente el uso de distribuciones a priori difusas o no informativas (Bilancia y Fedespina, 2009). Finalmente, el modelo BYM busca estimar la distribución de probabilidad a posteriori, de la cual se obtiene un estimador central (comúnmente la media o la mediana) y un intervalo de credibilidad.

Este modelo permite también la incorporación de covariables explicativas o de control, adoptando la forma:

$$
\log \theta_{i}=\alpha+\beta x_{i}+v_{i}+\varepsilon_{i}
$$

donde $x_{i}$ es el vector de covariables y $B$ es su coeficiente.

Una alternativa al modelo BYM, también dentro del enfoque jerárquico bayesiano, es la especificación Poisson-Gamma, que asigna una distribución gamma al componente aleatorio, e igualmente requiere la 
definición de distribuciones a priori para los hiperparámetros del modelo (Catelan, Lagazio y Biggeri, 2010).

En algunos eventos raros es posible una considerable cantidad de áreas sin casos observados (exceso de ceros), por lo que la distribución de los casos puede ser más dispersa que la distribución Poisson (sobredispersión). En estos casos existen dos métodos alternativos al modelo BYM: los modelos de mixturas finitas y el modelo mixto de riesgo relativo (Lawson y Clark, 2002; Lertxundi-Manterola, 2006).

\section{APLICACIONES}

A pesar de su menor capacidad para explicar causalidad, los estudios epidemiológicos espaciales con diseño ecológico han jugado un papel importante en la comprensión de los orígenes y las causas de las enfermedades, principalmente mediante el estudio de su distribución geográfica y el establecimiento de relaciones entre patrones geográficos y potenciales riesgos ambientales, dando origen a hipótesis etiológicas que han sido el punto de partida para la realización de otras investigaciones.

Los diseños multinivel desarrollados en las últimas décadas ofrecen la posibilidad de llevar a cabo estudios "semiecológicos" que incorporan variables medidas a nivel individual -como las relacionadas a los desenlaces y a las variables de confusión (edad, sexo, nivel socioeconómico, escolaridad, tipo de aseguramiento en salud, lugar de residencia al momento del diagnóstico, antecedentes ocupacionales, etc.)- con variables medidas en uno o más niveles de agregación -como, por ejemplo, las típicas mediciones o modelamientos de concentraciones de contaminantes atmosféricos en determinadas áreas geográficas(Elliott y Wartenberg, 2004; Wakefield, 2008) Los modelos bayesianos multinivel se han popularizado como una herramienta robusta y útil para suavizar o estabilizar estimaciones en áreas pequeñas (Beale et al., 2008). En la Tabla 1 se resumen algunos ejemplos de estudios ecológicos en áreas pequeñas que analizaron potenciales efectos de la contaminación ambiental sobre la morbilidad o la mortalidad por varias causas (general, cardiovascular, respiratoria y por cáncer), considerando diferentes definiciones de exposición y diferentes modelos estadísticos. 
Tabla 1. Estudios ecológicos en áress pequeñas sobre contaminación ambiental y efectos en salud

\begin{tabular}{|c|c|c|c|c|c|c|}
\hline Fatserenels & Luger & 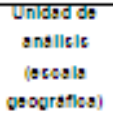 & $\begin{array}{l}\text { Definielon de } \\
\text { expocielon } \\
\text { smbiental }\end{array}$ & $\begin{array}{l}\text { Decenlece } \\
\text { on celud }\end{array}$ & Mods $1 \mathrm{am} / \mathrm{sib}$ & Rescultsdov/Consluetion \\
\hline $\begin{array}{l}\text { Ellomets. } \\
\text { (1998) }\end{array}$ & 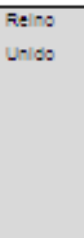 & $200 \mathrm{~s}$ ocsts & 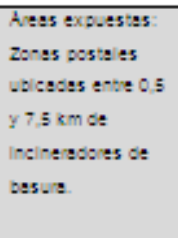 & $\begin{array}{l}\text { melomes } \\
\text { se cense }\end{array}$ & 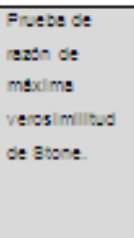 & 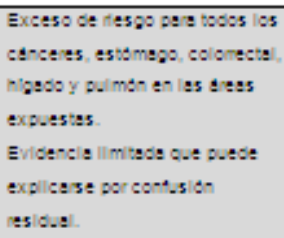 \\
\hline $\begin{array}{l}\text { Wiringon et: } \\
\text { (1997) }\end{array}$ & $\begin{array}{l}\text { Feino } \\
\text { uniso }\end{array}$ & $\begin{array}{l}\text { Bepmento } \\
\text { cense }\end{array}$ & 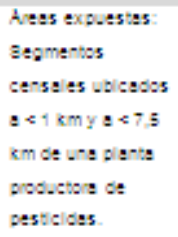 & $\begin{array}{l}\text { meidencley } \\
\text { motelded } \\
\text { oorcterer }\end{array}$ & 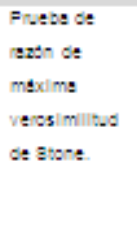 & 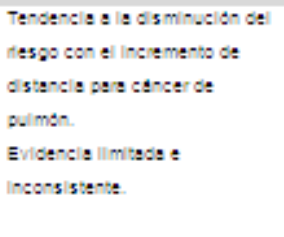 \\
\hline $\begin{array}{l}\text { Feynalds es: } \\
\text { (2000) }\end{array}$ & $\begin{array}{l}\text { ce tam } \\
\text { is } \\
\text { a uu }\end{array}$ & $\begin{array}{l}\text { Eopes } \\
\text { cense }\end{array}$ & 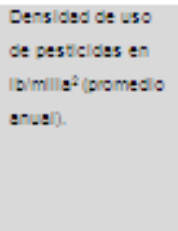 & 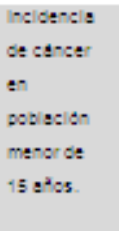 & $\begin{array}{l}\text { Feges ton } \\
\text { Feisson } \\
\text { etsice. }\end{array}$ & 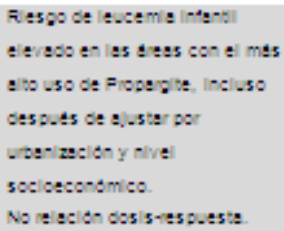 \\
\hline
\end{tabular}

Haga clic sobre la imagen para ampliarla

\section{DISCUSIÓN Y CONCLUSIONES}

La industria moderna usa miles de sustancias químicas sobre las cuales aún no se han realizado pruebas de su potencial toxicológico y se conoce poco respecto de su presencia y comportamiento en el ambiente, en especial de las mezclas de sustancias y de exposiciones en períodos críticos del desarrollo o en poblaciones específicas como niños, mujeres o ancianos (Boyle y Levin, 2008). Algunos de esos contaminantes están concentrados en áreas geográficas específicas, con importantes disparidades en la exposición, en especial en los países de reciente industrialización, donde las regulaciones suelen ser menos exigentes, y la contaminación ambiental suele afectar en mayor medida a las poblaciones de menor estatus socioeconómico (Norton et al., 2007), como es el caso de las denominadas "Villas de Cáncer" en las provincias de Henan y Jiangsu en China (Gao, 2013).

En muchos países, la disponibilidad de datos sobre eventos en salud está determinada por aspectos de confidencialidad respecto de información sensible, configurándose una tensión constante entre el derecho a la privacidad y el derecho a la democratización de la información (Jacquez, 2004). Los datos disponibles habitualmente corresponden a datos agregados en áreas político-administrativas que en muchos casos no guardan correspondencia espacial ni temporal con los datos sobre exposiciones ambientales (Jacquez, 2004). En los países en desarrollo, la información disponible se limita a las defunciones en la mayoría de los casos, junto a los registros de egreso hospitalario, en algunos otros; la cobertura y la calidad de esta información es, sin duda, un factor importante. Colombia ha logrado importantes avances en la recopilación y 
sistematización de información sobre eventos en salud, aunque en algunas zonas del país el subregistro sigue siendo un problema por resolver.

Por su parte, la disponibilidad de datos sobre contaminantes ambientales es una limitación mayor, en especial porque que los datos sobre exposiciones ambientales suelen ser datos sustitutos o proxies de las verdaderas exposiciones (Jacquez, 2004; Beale et al., 2008). No obstante, el uso de variables sustitutas de exposición está justificado por la dificultad -o imposibilidad- de contar con mediciones precisas de exposición. La disponibilidad de datos públicos sobre contaminantes descargados al ambiente es un tema que amerita mayor atención por parte de las autoridades, en especial en los países en desarrollo. En Colombia, las mediciones de contaminantes ambientales tienen menos de dos décadas y su cobertura y desagregación geográfica no permiten llevar a cabo análisis rutinarios en áreas más pequeñas que el nivel municipal. Sin embargo, destacan los esfuerzos realizados por investigadores en algunas ciudades del país, donde se han adelantado mediciones de contaminantes ambientales y estudios epidemiológicos serios sobre los efectos de la contaminación ambiental en la salud de las poblaciones (Filigrana, Méndez y Gómez, 2008; Ocampo, Pradilla y Méndez, 2008; Filigrana y Méndez, 2012).

Si bien hay una preocupación creciente por los temas ambientales, es necesario continuar desarrollando sistemas estandarizados de medición y monitoreo de contaminación del aire, del agua, del suelo y de los alimentos, y lograr una adecuada difusión y utilización de la información recopilada. Las técnicas de modelamiento espacial de exposiciones ambientales son una herramienta útil para optimizar la información disponible sobre contaminación ambiental.

Diversos estudios epidemiológicos han señalado asociaciones entre exposición a contaminantes ambientales y la aparición de efectos deletéreos en salud humana, que van desde la exacerbación de síntomas respiratorios en niños asmáticos (Escamilla-Núñez et al., 2008) hasta incrementos en las tasas de mortalidad general y por causas específicas (Filleul et al., 2005; Lepeule, Laden, Dockery y Schwartz, 2012; Heinrich et al., 2013). Muchos de esos estudios han utilizado técnicas de análisis espacial para estimar o modelar exposiciones ambientales, o para ajustar y suavizar tasas en áreas pequeñas afectadas por contaminación, como por ejemplo los estudios que relacionan diversas exposiciones con aumentos en la incidencia y la mortalidad por cáncer (Katsouyanni y Pershagen, 1997; Boffetta y Nyberg, 2003; Nafstad et al., 2003; Knox, 2005a, 2005b; Zambon et al., 2007; Carozza et al., 2008; Clapp, Jacobs y Loechler, 2008; Carozza, Li, Wang, Horel y Cooper, 2009; Ramis et al., 2009, 2012; Roos et al., 2010; López-Abente et al., 2012; RaaschouNielsen et al., 2013).

En lo que respecta a los diseños metodológicos, es importante tener en cuenta las limitaciones de los análisis con datos agregados, en particular en lo referente al sesgo ecológico, y a la falta de un adecuado control de variables confusoras o modificadoras de efecto, sobre todo por la poca información disponible sobre tales variables. Este tipo de estudios da cuenta de asociaciones epidemiológicas pero no puede por sí solo demostrar causalidad de manera inequívoca. 
Sin embargo, expertos en el tema consideran que usar áreas pequeñas tiene ventajas respecto del uso de áreas geográficas mayores, en tanto los estudios a pequeña escala son menos susceptibles al componente del sesgo ecológico dado por la variabilidad intra-área, por lo que es posible encontrar mayor homogeneidad respecto de algunas exposiciones y variables confusoras a nivel poblacional (Elliott y Wartenberg, 2004). Además, los resultados de estos estudios son más fácilmente interpretables y tienen mayor capacidad de detectar efectos altamente localizados como los relacionados a la contaminación industrial (Richardson, Thomson, Best y Elliott, 2004). El análisis espacial ofrece la oportunidad de optimizar el uso de datos generados rutinariamente, mediante la aplicación de técnicas estadísticas sofisticadas como los modelos multinivel, que permiten la combinación de datos individuales con datos agregados sobre "variables contextuales" que afectan a grupos de individuos y no pueden ser medidas a nivel individual (Jacquez, 2004).

En los estudios en áreas pequeñas, los errores y los sesgos tienden a producir resultados negativos más que positivos. Por el contrario, los confusores pueden actuar en sentido opuesto señalando asociaciones positivas entre contaminación ambiental y eventos en salud en ausencia de un vínculo causal (Olsen et al., 1996). No obstante, es destacable que los análisis espaciales en áreas pequeñas pueden disminuir o incluso eliminar el sesgo ecológico al reducir la variabilidad de las exposiciones al interior de una misma área, como por ejemplo, un distrito censal (Lertxundi-Manterola, 2006).

Rothmann et al. señalan que los diseños ecológicos son útiles para realizar hypothesis screening dado que, si bien su validez y precisión son inferiores a los de otros diseños, resultan fáciles y menos costosos para probar inicialmente una posible asociación entre una exposición y un efecto en salud, que justifique la realización de estudios confirmatorios más rigurosos, con mejores diseños y mayores costos (Rothman et al., 2008).

Los estudios ecológicos en áreas pequeñas también pueden ser utilizados en vigilancia epidemiológica para resaltar áreas con posible riesgo elevado, permitiendo que las autoridades actúen proactivamente, no solo en lo relacionado con la investigación etiológica, sino también en la identificación precoz de necesidades y en la solución de otros asuntos de interés público, como los servicios asistenciales o la respuesta a alarmas sanitarias (Santamaría-Ulloa, 2003; Elliott y Wartenberg, 2004). Vigilar la agregación espacial de eventos en salud pública facilita el direccionamiento de intervenciones dirigidas a controlar causas o factores de riesgo ya conocidos para ese evento. $\mathrm{O}$, en dirección contraria, permite también vigilar el patrón espacial de factores de riesgo con el fin de anticipar y prevenir la ocurrencia de conglomerados de casos mediante medidas de salud pública dirigidas a remover o reducir el efecto de ese factor de riesgo sobre la población general (Lawson et al., 1999), tal y como operan las alertas por contaminación atmosférica cuando se superan los niveles máximos permisibles de contaminantes.

Los avances de las ciencias computacionales y la masificación de los dispositivos móviles, capaces de capturar y transmitir datos espaciales en tiempo real, aparecen como una tremenda oportunidad para la generación de STIS (Spatio Temporal Information Systems), muy superiores al análisis estático que ofrecen los GIS (Geographical Information Systems), con los cuales se hace posible analizar espacio-temporalmente 
exposiciones ambientales y problemas de salud asociados (Jacquez, 2004), incorporando información crucial como la relativa a los desplazamientos de las personas y las exposiciones en diferentes ambientes como el domicilio, el sitio de trabajo, los medios de transporte, las áreas recreativas, entre otras. La existencia de datos puntuales a partir de registros especializados (cáncer, malformaciones congénitas, eventos cardiovasculares, etc.) amplía las posibilidades de análisis y ofrece la posibilidad de probar hipótesis causales con mayor solidez.

\section{CONFLICTO DE INTERESES}

El autor declara no tener ningún conflicto de intereses.

\section{AGRADECIMIENTOS}

Al Dr. Pablo Ruiz Rudolph, académico de la Universidad de Chile, por su lectura y comentarios para el mejoramiento de este manuscrito.

\section{REFERENCIAS}

- Abrahams, N., Lasso, A., Filigrana, P., Silva, J., y Méndez, F. (2009). PM2.5 and Heavy Metals Outdoor Concentrations at a School Near to an Industrial Zone in Cali, Colombia. Epidemiology, 20(6), S69-S70.

- Agencia Europea de Medio Ambiente. (2013). Registro Europeo de Emisiones y Tranferencias de Contaminantes (E-PRTR). Recuperado de http://prtr.ec.europa.eu/pgAbout.aspx

- Beale, L., Abellán, J. J., Hodgson, S., y Jarup, L. (2008). Methodologic issues and approaches to spatial epidemiology. Environmental Health Perspectives, 116(8), 1105-10. doi: 10.1289/ehp.10816.

- Belpomme, D., Irigaray, P., Hardell, L., Clapp, R., Montagnier, L., Epstein, S., y Sasco, A. J. (2007). The multitude and diversity of environmental carcinogens. Environmental Research, 105(3), 414-429.

- Bilancia, M., y Fedespina, A. (2009). Geographical clustering of lung cancer in the province of Lecce, Italy: 1992-2001. International Journal of Health Geographics, 8, 40. doi: 10.1186/1476-072X-8-40.

- Boffetta, P., y Nyberg, F. (2003). Contribution of environmental factors to cancer risk. British Medical Bulletin, 68, 71-94. doi: 10.1093/bmb/ldg023.

- Boyle, P., y Levin, B. (2008). World cancer report. (P. Boyle \& B. Levin, Eds.). Lyon: International Agency for Research on Cancer.

- Briggs, D. (2000). Exposure assessment. En P. Elliott, J. Wakefield, N. Best, y D. Briggs (Eds.), Spatial epidemiology: methods and applications (pp. 335-355). New York: Oxford University Press.

- Carozza, S. E., Li, B., Elgethun, K., y Whitworth, R. (2008). Risk of Childhood Cancers Associated with Residence in Agriculturally Intense Areas in the United States. Environmental Health Perspectives, 116(4), 559-565.

- Carozza, S. E., Li, B., Wang, Q., Horel, S., y Cooper, S. (2009). Agricultural pesticides and risk of childhood cancers. International Journal of Hygiene and Environmental Health, 212(2), 186-195. 
- Catelan, D., Lagazio, C., y Biggeri, A. (2010). A hierarchical Bayesian approach to multiple testing in disease mapping. Biometrical Journal, 52(6), 784-97. doi: 10.1002/bimj.200900209.

- Centers for Disease Control and Prevention. (1990). Guidelines for investigating clusters of disease events. Recommendations and reports (RR-11). MMWR (pp. 1-23). Atlanta.

- Clapp, R., Jacobs, M., y Loechler, E. (2008). Environmental and occupational causes of cancer. New evidence, 2005-2007. Reviews on Environmental Health, 23(1), 1-37.

- Cogliano, V. J., Baan, R., Straif, K., Grosse, Y., Lauby-Secretan, B., El Ghissassi, F., ... Wild, C. P. (2011). Preventable exposures associated with human cancers. Journal of the National Cancer Institute, 103(24), 1827-39. doi: 10.1093/jnci/djr483.

- Colvile, R., Briggs, D., y Nieuwenhuijsen, M. (2003). Environmental measurement and modelling: introduction and source dispersion modelling. En M. J. Nieuwenhuijsen (Ed.), Exposure assessment in occupational and environmental epidemiology (pp. 39-53). New York: Oxford University Press.

- Correal-Arboleda, E., y Gamboa-Salazar, J. (2006). Inventario de Emisiones Atmosféricas del Municipio de Montería. Tesis de Grado. Facultad de Ingenierías, Universidad de Medellín.

- Cortés, S., y Qüense, J. (2014). Uso de herramientas espaciales para el análisis de la información sanitaria-ambiental en comunidades vulnerables. En Propuestas para Chile. Concurso de Políticas Públicas 2013 (pp. 221240). Santiago de Chile: Pontificia Universidad Católica de Chile.

- Cuzick, J., y Elliott, P. (1992). Small-area studies: purpose and methods. En P. Elliot, J. Cuzick, D. English, y R. Stern (Eds.), Geographical and Environmental Epidemiology: methods for small-area studies (pp. 14-21). New York: Oxford University Press.

- Edwards, R., Pless-Mulloli, T., Howel, D., Chadwick, T., Bhopal, R., Harrison, R., y Gribbin, H. (2006). Does living near heavy industry cause lung cancer in women? A case-control study using life grid interviews. Thorax, 61(12), 1076-82. doi: 10.1136/thx.2005.057620.

- Elliott, P. (1995). Investigation of disease risks in small areas. Occupational and Environmental Medicine, 52(12), 785-9.

- Elliott, P., Shaddick, G., Kleinschmidt, I., Jolley, D., Walls, P., Beresford, J., y Grundy, C. (1996). Cancer incidence near municipal solid waste incinerators in Great Britain. British Journal of Cancer, 73(5), 702-710.

- Elliott, P., Shaddick, G., Wakefield, J. C., de Hoogh, C., y Briggs, D. J. (2007). Long-term associations of outdoor air pollution with mortality in Great Britain. Thorax, 62(12), 1088-94. doi: 10.1136/thx.2006.076851.

- Elliott, P., y Wartenberg, D. (2004). Spatial epidemiology: current approaches and future challenges. Environ Health Perspect, 112(9), 9981006.

- EPA. (2013). United States Environmental Protection Agency. Toxics Release Inventory (TRI) program. Recuperado de http://www2.epa.gov/toxics-release-inventory-tri-program/learn-abouttoxics-release-inventory

- Escamilla-Núñez, M.-C., Barraza-Villarreal, A., Hernández-Cadena, L., Moreno-Macías, H., Ramírez-Aguilar, M., Sienra-Monge, J.-J., ... Romieu, I. (2008). Traffic-related air pollution and respiratory symptoms among asthmatic children, resident in Mexico City: the EVA cohort study. Respiratory Research, 9(1), 74.

- Feja-Solana, C., Alcalá-Nalvaiz, J. T., Rabanaque-Hernández, M. J., SaezSafra, M., Marcos-Grágera, R., y Martos-Jiménez, M. C. (2010). Desigualdades geográficas en mortalidad e incidencia por cáncer de 
laringe en varones: factores socioeconómicos y ambientales. Rev Esp Salud Pública, 84(6), 745-756.

- Filigrana, P., Jiménez, L., Abrahams, N., Cuartas, D., y Méndez, F. (2011). Association between particulate matter (PM), O3 and NOx and emergency room (ER) visitis and hospital admission for respiratory and cardiovascular diseases in Cali-Colombia. En Annual Conference of the International Society for Environmental Epidemiology - ISEE.

- Filigrana, P., y Méndez, F. (2012). Blood lead levels in schoolchildren living near an industrial zone in Cali, Colombia: the role of socioeconomic condition. Biol Trace Elem Res, 149(3), 299-306.

- Filigrana, P., Méndez, F., y Gómez, R. (2008). Descripción de la calidad del aire en el área de influencia del botadero de Navarro, Cali, Colombia. Colomb. Méd., 39, 245-252.

- Filleul, L., Rondeau, V., Vandentorren, S., Le Moual, N., Cantagrel, A., Annesi-Maesano, I., ... Baldi, I. (2005). Twenty five year mortality and air pollution: results from the French PAARC survey. Occupational and Environmental Medicine, 62(7), 453-60. doi: 10.1136/oem.2004.014746.

- Fortunato, L., Abellán, J. J., Beale, L., LeFevre, S., y Richardson, S. (2011). Spatio-temporal patterns of bladder cancer incidence in Utah (1973-2004) and their association with the presence of toxic release inventory sites. International Journal of Health Geographics, 10(1), 16. doi: 10.1186/1476-072X-10-16.

- Gao, H. (2013). Chinese Government admits existence of cancer villages. The Lancet Oncology, 14(4), 284. doi: 10.1016/S14702045(13)70065-2.

- García-Pérez, J., Boldo, E., Ramis, R., Pollán, M., Pérez-Gómez, B., Aragonés, N., y López-Abente, G. (2007). Description of industrial pollution in Spain. BMC Public Health, 7, 40.

- Guajardo, O. A., y Oyana, T. J. (2009). A critical assessment of geographic clusters of breast and lung cancer incidences among residents living near the Tittabawassee and Saginaw Rivers, Michigan, USA. Journal of Environmental and Public Health, ID 316249. doi: 10.1155/2009/316249.

- Heinrich, J., Thiering, E., Rzehak, P., Krämer, U., Hochadel, M., Rauchfuss, K. M., ... Wichmann, H.-E. (2013). Long-term exposure to NO2 and PM10 and all-cause and cause-specific mortality in a prospective cohort of women. Occupational and Environmental Medicine, 70(3), 17986. doi: 10.1136/oemed-2012-100876.

- Hendryx, M., y Ahern, M. M. (2008). Relations between health indicators and residential proximity to coal mining in West Virginia. American Journal of Public Health, 98(4), 669-71. doi: 10.2105/AJPH.2007.113472.

- Holowaty, E. J., Norwood, T. A., Wanigaratne, S., Abellán, J. J., y Beale, L. (2010). Feasibility and utility of mapping disease risk at the neighbourhood level within a Canadian public health unit: an ecological study. International Journal of Health Geographics, 9, 21. doi: 10.1186/1476-072X-9-21.

- Hystad, P., Demers, P. A., Johnson, K. C., Brook, J., van Donkelaar, A., Lamsal, L., ... Brauer, M. (2012). Spatiotemporal air pollution exposure assessment for a Canadian population-based lung cancer case-control study. Environmental Health, 11(1), 22. doi: 10.1186/1476-069X-11-22.

- INECC-SERMANAT. (2005). Guía de elaboración y usos de inventarios de emisiones . Instituto Nacional de Ecología y Cambio Climático - Secretaría de Medio Amiente y Recursos Naturales, México D.F.

- Jacquez, G. M. (2004). Current practices in the spatial analysis of cancer: flies in the ointment. International Journal of Health Geographics, 3(1), 22.

- Katsouyanni, K., y Pershagen, G. (1997). Ambient air pollution exposure and cancer. Cancer Causes \& Control, 8(3), 284-91. 
- Knox, E. G. (2005a). Childhood cancers and atmospheric carcinogens. Journal of Epidemiology \& Community Health, 59(2), 101105.

- Knox, E. G. (2005b). Oil combustion and childhood cancers. Journal of Epidemiology \& Community Health, 59(9), 755-760.

- Krieger, N., Chen, J. T., Waterman, P. D., Soobader, M.-J., Subramanian, S. V., y Carson, R . (2002). Geocoding and monitoring of US socioeconomic inequalities in mortality and cancer incidence: Does the choice of area-based measure and geographic level matter?: The Public Health Disparities Geocoding Project. American Journal of Epidemiology, 156(5), 471-482. doi: 10.1093/aje/kwf068.

- Lawson, A. B., Browne, W. J., y Vidal Rodeiro, C. L. (2003). Disease Mapping with WinBUGS and MLwiN. Chichester, England: Wiley \& Sons Ltd.

- Lawson, A. B., y Clark, A. (2002). Spatial mixture relative risk models applied to disease mapping. Statistics in Medicine, 370 (April 2001), 359370. doi: 10.1002/sim.1022.

- Lawson, A., Biggeri, A., Böhning, D., Lesaffre, E., Viel, J., y Bertollini, R. (1999). Disease mapping and risk assessment in public health. (A. Lawson, A. Biggeri, D. Böhning, E. Lesaffre, J. Viel, y R. Bertollini, Eds.). Chichester: John Wiley \& Sons.

- Lepeule, J., Laden, F., Dockery, D., y Schwartz, J. (2012). Chronic exposure to fine particles and mortality: an extended follow-up of the Harvard Six Cities study from 1974 to 2009. Environmental Health Perspectives, 120(7), 965-70. doi: 10.1289/ehp.1104660.

- Lertxundi-Manterola, A. (2006). Métodos de estadística espacial para evaluar la influencia de factores medioambientales sobre la incidencia y mortalidad por cáncer. Tesis Doctoral. Universitat de Girona.

- Liaw, J., Marshall, G., Yuan, Y., Ferreccio, C., Steinmaus, C., y Smith, A. $H$. (2008). Increased childhood liver cancer mortality and arsenic in drinking water in northern Chile. Cancer Epidemiology, Biomarkers \& Prevention, 17(8), 1982-7. doi: 10.1158/1055-9965.EPI-07-2816.

- López-Abente, G., García-Pérez, J., Fernández-Navarro, P., Boldo, E., y Ramis, R. (2012). Colorectal cancer mortality and industrial pollution in Spain. BMC Public Health, 12, 589. doi: 10.1186/1471-2458-12-589.

- Marshall, G., Ferreccio, C., Yuan, Y., Bates, M. N., Steinmaus, C., Selvin, S., ... Smith, A. H. (2007). Fifty-year study of lung and bladder cancer mortality in Chile related to arsenic in drinking water. $J$ Nat Cancer Inst, 99(12), 920-8. doi: 10.1093/jnci/djm004.

- Ministerio de Ambiente Vivienda y Desarrollo Territorial (Colombia). (2005). Decreto 4741 de 2005. Por el cual se reglamenta parcialmente la prevención y manejo de los residuos o desechos peligrosos generados en el marco de la gestión integral. Bogotá. El Ministerio.

- Ministerio de Ambiente Vivienda y Desarrollo Territorial (Colombia). (2010). Protocolo para el monitoreo y seguimiento de la calidad del aire Manual de operacion de sistemas de vigilancia de la calidad del aire. Bogotá: El Ministerio.

- Ministerio de Ambiente y Desarrollo Sostenible (Colombia). (2014). Registro de Generadores de Residuos Peligrosos. Sitio web del Sistema de Información Ambiental de Colombia. Recuperado de https://www.siac.gov.co/contenido/contenido.aspx?conID=540\&catID=4 $\underline{16}$

- $\quad$ Ministerio de Salud (Colombia). (2015). Sistema Integral de Información de la Protección Social. Sitio web. Recuperado de http://www.sispro.gov.co/Pages/ContruyaSuConsulta/Aseguramiento.as $\mathrm{px}$ 
- Monge-Corella, S., García-Pérez, J., Aragonés, N., Pollán, M., PérezGómez, B., y López-Abente, G. (2008). Lung cancer mortality in towns near paper, pulp and board industries in Spain: a point source pollution study. BMC Public Health, 8, 288-99. doi: 10.1186/1471-2458-8-288.

- Morris, S., y Wakefield, J. (2000). Assessment of disease risk in relation to a pre-specified source. En P. Elliot, J. Wakefield, N. Best, \& D. Briggs (Eds.), Spatial epidemiology: methods and applications (1a ed., pp. 153184). New York: Oxford University Press.

- Nafstad, P., Håheim, L. L., Oftedal, B., Gram, F., Holme, I., Hjermann, I., y Leren, P. (2003). Lung cancer and air pollution: a 27 year follow up of 16 209 Norwegian men. Thorax, 58(12), 1071-6.

- Nazaroff, W., y Álvarez-Cohen, L. (2001). Environmental Engineering Science, (1a ed.). New York: John Wiley \& Sons.

- Norton, J. M., Wing, S., Lipscomb, H. J., Kaufman, J. S., Marshall, S. W., y Cravey, A. J. (2007). Race, wealth, and solid waste facilities in North Carolina. Environmental Health Perspectives, 115(9), 1344-50. doi: 10.1289/ehp.10161.

- Nuvolone, D., Della Maggiore, R., Maio, S., Fresco, R., Baldacci, S., Carrozzi, L., ... Viegi, G. (2011). Geographical information system and environmental epidemiology: a cross-sectional spatial analysis of the effects of traffic-related air pollution on population respiratory health. Environmental Health, 10(1), 12. doi: 10.1186/1476-069X-10-12.

- Ocampo, C., Pradilla, A., y Méndez, F. (2008). Impacto de un depósito de residuos sólidos en el crecimiento físico infantil. Colomb. Méd., 39, 253259.

- Olsen, S., Martuzzi, M., y Elliott, P. (1996). Cluster analysis and disease mapping: why, when and how. A step by step guide. $\mathrm{Br}$ Med J, 313, 863. doi: http://dx.doi.org/10.1136/bmj.313.7061.863

- Peñaloza, N. (2010). Distribución espacial y temporal del inventario de emisiones provenientes de las fuentes móviles y fijas de la ciudad de Bogotá, D.C. Tesis de Maestría. Departamento de Ingeniería Química y Ambiental. Universidad Nacional de Colombia.

- Puigpinós-Riera, R., Marí-Dell'Olmo, M., Gotsens, M., Borrell, C., Serral, G., Ascaso, C., ... Sánchez-Villegas, P. (2011). Cancer mortality inequalities in urban areas: a Bayesian small area analysis in Spanish cities. International Journal of Health Geographics, 10(1), 6. doi: 10.1186/1476-072X-10-6.

- Putila, J. J., y Guo, N. L. (2011). Association of arsenic exposure with lung cancer incidence rates in the United States. PloS One, 6(10), e25886. doi: 10.1371/journal.pone.0025886.

- Raaschou-Nielsen, O., Andersen, Z. J., Beelen, R., Samoli, E., Stafoggia, M., Weinmayr, G., ... Hoek, G. (2013). Air pollution and lung cancer incidence in 17 European cohorts: prospective analyses from the European Study of Cohorts for Air Pollution Effects (ESCAPE). The Lancet Oncology, 14(9), 813-22. doi: 10.1016/S1470-2045(13)70279-1.

- Ramis, R., Fernández-Navarro, P., García-Pérez, J., Boldo, E., GómezBarroso, D., y López-Abente, G. (2012). Risk of cancer mortality in spanish towns lying in the vicinity of pollutant industries. ISRN Oncology, 614198. doi: 10.5402/2012/614198.

- Ramis, R., Vidal, E., García-Pérez, J., Lope, V., Aragonés, N., PérezGómez, B., ... López-Abente, G. (2009). Study of non-Hodgkin's lymphoma mortality associated with industrial pollution in Spain, using Poisson models. BMC Public Health, 9, 26. doi: 10.1186/1471-2458-9-26.

- Reynolds, P., Von Behren, J., Gunier, R. B., Goldberg, D. E., Hertz, A., y Harnly, M. E. (2002). Childhood cancer and agricultural pesticide use: an ecologic study in California. Environ Health Perspect, 110(3), 319-24. 
- Richardson, S., Thomson, A., Best, N., y Elliott, P. (2004). Interpreting posterior relative risk estimates in disease-mapping studies. Environmental Health Perspectives, 112(9), 1016-1025. doi: 10.1289/ehp.6740.

- Roos, A. J. De, Davis, S., Colt, J. S., Blair, A., Airola, M., Severson, R. K., ... Ward, M. (2010). Residential proximity to industrial facilities and risk of non-Hodgkin lymphoma. Environmental Research, 110(1), 70-78. doi: 10.1016/j.envres.2009.09.011.

- Rothman, K., Greenland, S., y Lash, T. (2008). Modern epidemiology, (Third edit.). Philadelphia: Lippincott Williams \& Wilkins.

- Sahsuvaroglu, T., Jerrett, M., Sears, M. R., McConnell, R., Finkelstein, N., Arain, A., ... Burnett, R. (2009). Spatial analysis of air pollution and childhood asthma in Hamilton, Canada: comparing exposure methods in sensitive subgroups. Environmental Health, 8(2), 14. doi: 10.1186/1476069X-8-14.

- Salinas, A. (2007). Organización de Estados Americanos. Preparación de Registros de Emisión y Transferencia de Contaminantes (RETC) en América Latina $y$ el Caribe. Recuperado de http://www.oas.org/dsd/Quimicos/RegistrosdeEmisiónyTransferenciade Contaminantes.pdf

- Santamaría-Ulloa, C. (2003). Evaluación de alarmas por cáncer utilizando análisis espacial: una aplicación para Costa Rica. Revista Costarricense de Salud Pública, 12(21), 18-22.

- Tseng, C.-Y., Huang, Y.-C., Su, S.-Y., Huang, J.-Y., Lai, C.-H., Lung, C.C., ... Liaw, Y.-P. (2012). Cell type specificity of female lung cancer associated with sulfur dioxide from air pollutants in Taiwan: an ecological study. BMC Public Health, 12(1), 4. doi: 10.1186/1471-2458-12-4.

- United States Government Accountability Office. (2005). Chemical regulation. Options Exist to Improve EPA's Ability to Assess Health Risks and Manage Its Chemical Review Program. GAO-05-458 Report to Congressional Requesters. Washington, D.C.

- Universidad Pontificia Bolivariana. (2010). Evaluación de los niveles de contaminación atmosférica en las zonas urbanas del Valle de Aburrá. Medellín.

- Vinikoor-Imler, L. C., Davis, J. A., y Luben, T. J. (2011). An ecologic analysis of county-level PM2.5 concentrations and lung cancer incidence and mortality. International Journal of Environmental Research and Public Health, 8(6), 1865-71. doi: 10.3390/ijerph8061865.

- Wakefield, J. (2008). Ecologic studies revisited. Annual Review of Public Health, 29, 75-90. doi: 10.1146/annurev.publhealth.29.020907.090821.

- Waller, L. A., y Gotway, C. A. (2004). Applied spatial statistics for public health data. New Jersey: Wiley-Interscience. John Wiley \& Sons, Inc. doi: 10.1198/jasa.2005.s15.

- Wheeler, D. C., Ward, M. H., y Waller, L. A. (2012). Spatial-temporal analysis of cancer risk in epidemiologic studies with residential histories. Ann Assoc Am Geogr, 102(5), 1049-1057. doi: 10.1080/00045608.2012.671131.

- Wilkinson, P., y Armstrong, B. (2006). Analysis and interpretation of a single-site cluster. En P. Wilkinson (Ed.), Environmental Epidemiology (pp. 27-40). Berkshire, England: Open University Press - McGraw-Hill Education.

- Wilkinson, P., Thakrar, B., Shaddick, G., Stevenson, S., Pattenden, S., Landon, M., ... Elliott, P. (1997). Cancer incidence and mortality around the Pan Britannica Industries pesticide factory, Waltham Abbey. Occupational and Environmental Medicine, 54(2), 101-7.

- Zambon, P., Ricci, P., Bovo, E., Casula, A., Gattolin, M., Fiore, A. R., ... Guzzinati, S. (2007). Sarcoma risk and dioxin emissions from incinerators 
and industrial plants: a population-based case-control study (Italy). Environmental Health, 6(1), 19.

1. Médico Cirujano. Docente Departamento de Salud Pública, Universidad de Caldas. Candidato a Doctor en Salud Pública, Universidad de Chile. nelson.arias@ucaldas.edu.co

2. Traducción del autor.

3. Habitualmente se estratifica por sexo, edad, año calendario y condición socioeconómica, aunque deben incorporarse otras variables confusoras si existe información disponible.

Para citar este artículo: Arias-Ortiz, N. E. (2016). Estudios epidemiológicos en áreas pequeñas: herramientas para analizar la contaminación ambiental y sus efectos en salud a escala local. Revista Luna Azul, 42, 341-361. Recuperado de http://200.21.104.25/lunazul/index.php?option=com_content\&view=artic le\&id=142 